\title{
An in vitro Mechanical Damage Model of Isolated Myofibers in a Floating Culture Condition
}

Yoshifumi Tsuchiya and Yusuke Ono*

Department of Muscle Development and Regeneration, Institute of Molecular Embryology and Genetics, Kumamoto University, 2-2-1 Honjo, Kumamoto 860-0811, Japan

*For correspondence: ono-y@kumamoto-u.ac.jp

[Abstract] Muscle stem cells (satellite cells), located on the surface of myofibers, are rapidly activated from a quiescent state following skeletal muscle injury. Although satellite cell activation is an initial step in muscle regeneration, the stimulation of satellite cell activation by muscle injury remains to be elucidated. We recently established an in vitro mechanical damage model of myofibers, to analyze quiescent and activated satellite cells associated with myofibers isolated from the extensor digitorum longus muscle in mice. Here, we described a protocol for the mechanical damage of myofibers and coculture of intact healthy myofibers with damaged myofibers in a floating condition. This in vitro myofiber damage model allowed us to investigate the mechanism of satellite cell activation without contamination by interstitial cells, such as blood vessel cells and fibroblasts, as well as understand how damaged myofiber-derived factors (DMDFs) activate satellite cells.

Keywords: Skeletal muscle, Satellite cells, Muscle stem cells, Muscle injury, Muscle regeneration, Myokine, Damaged myofiber-derived factors

[Background] Muscle stem cells called satellite cells reside between the plasmalemma of myofibers and the basal lamina, and maintain a quiescent state in healthy muscles (Mauro, 1961). Satellite cells play important roles in postnatal muscle growth, hypertrophy, and regeneration, by providing new myonuclei of myofibers in adults. Following muscle injury, satellite cells are rapidly activated from their quiescent state to become myoblasts. Then, they proliferate to give rise to progeny, and fuse with one another and/or with existing damaged myofibers to regenerate muscles. Although the activation of satellite cells is an initial step in muscle regeneration, its mechanism remains to be elucidated.

Extracts from crushed muscle tissues were observed to stimulate the activation and proliferation of cultured myogenic cells (Vandenburgh et al., 1984; Kardami et al., 1985; Bischoff, 1986; Mezzogiorno et al., 1993; Haugk et al., 1995), indicating that muscle tissues contain an activator of satellite cells. Consequently, cytokines and growth factors, including hepatocyte growth factor, have been identified as activators of satellite cells (Tatsumi et al., 1998; Li, 2003; Zeng et al., 2010). Muscle tissues are composed of myofibers as well as various types of other cells, such as interstitial mesenchymal cells and immune cells (e.g., blood vessel cells, macrophages, and fibroblasts) (Evano and Tajbakhsh, 2018). Thus, such non-muscle cell-derived factors may directly and indirectly influence satellite cell dynamics and contribute to muscle regeneration.

The individual myofiber isolation and culture technique is a useful tool to analyze satellite cell fate dynamics, ranging from quiescence to activation, proliferation, differentiation, and self-renewal in their 
Please cite this article as: Tsuchiya, Y. and Ono, Y. (2022). An in vitro Mechanical Damage Model of Isolated Myofibers in a Floating Culture Condition

niche of myofibers, without contamination by interstitial cells (Zammit et al., 2004; Ono et al., 2011). By applying this culture system, we recently established an in vitro mechanical damage model of isolated myofibers in a floating culture condition. This model can be applied to the co-culture of intact myofibers harboring satellite cells with mechanically damaged myofibers, allowing us to examine how damaged myofiber-derived factors (DMDFs) influence satellite cell dynamics (Tsuchiya et al., 2020), and better understand the mechanism of the initial step of muscle regeneration. Here, we describe a detailed protocol for the isolation and mechanical damage of individual myofibers isolated from the extensor digitorum longus (EDL) muscle in mice, as well as floating co-culture of intact myofibers and damaged myofibers. Our in vitro mechanical myofiber damage model is useful for developing efficient regeneration therapies for muscle wasting diseases, such as muscular dystrophies and age-related sarcopenia, as well as for establishing a strategy for rapid recovery from severe muscle injury in athletes.

\section{Materials and Reagents}

\section{Materials}

1. C57BL/6 mice at $8-12$ weeks old

2. Glass Pasteur pipettes (IWAKI, catalog number: IK-PAS-5P, 200)

3. Diamond pen (AS ONE, catalog number: 6-539-05)

4. Rubber pipette bulbs

5. $7 \mathrm{~mL}$ Polystyrene Bijou Sample Containers (Sterilin, catalog number: 129A)

6. 6-well plates (Sanplatec, catalog number: 26510)

7. Deep Petri dishes $(\varphi 50 \times 20.3 \mathrm{~mm})$ (Sterilin catalog number: 124)

8. $25 \mathrm{~mL}$ self-standing tube (IWAKI, catalog number: 2362-025)

9. Sterilized empty bottle to throw out washing media

10. $0.45 \mu \mathrm{m}$ syringe filters (Sartorius, catalog number: 16533)

11. Slide-glass (Matsunami, catalog number: MAS-01)

12. Cover-glass $(24 \times 55 \mathrm{~mm})$ (Matsunami, catalog number: C024551)

13. Pap pen (Daido Sangyo, catalog number: S-PAP)

\section{Reagents}

1. Dulbecco's modified Eagle medium (DMEM) (Gibco, catalog number: 11995065, storage temperature: $4^{\circ} \mathrm{C}$ )

2. Penicillin and streptomycin (PS) solution $(\times 100)$ (Wako, catalog number: 168-23191, storage temperature: $4^{\circ} \mathrm{C}$ )

3. Dulbecco's phosphate-buffered saline (PBS) (Wako, catalog number: 045-29795, storage temperature: $\left.15-25^{\circ} \mathrm{C}\right)$

4. Bovine serum albumin (BSA) (Sigma, catalog number: A9418, storage temperature: $4^{\circ} \mathrm{C}$ )

5. Goat serum (Sigma, catalog number: G9023, storage temperature: $4^{\circ} \mathrm{C}$ ) 
Please cite this article as: Tsuchiya, Y. and Ono, Y. (2022). An in vitro Mechanical Damage Model of Isolated Myofibers in a Floating Culture Condition.

6. 4\%-Paraformaldehyde (PFA) solution (Nacalai Tesque, catalog number: 09154-85, storage temperature: $\left.4^{\circ} \mathrm{C}\right)$

7. Triton $\mathrm{X}-100$ (Sigma, catalog number: $\mathrm{T} 8787$, storage temperature: $15-25^{\circ} \mathrm{C}$ )

8. Tween 20 (Sigma, catalog number: P9416, storage temperature: $15-25^{\circ} \mathrm{C}$ )

9. 4',6-diamidino-2-phenylindole (DAPI) containing mounting medium (Nacalai tesque, catalog number: $12745-74$, storage temperature: $4^{\circ} \mathrm{C}$ )

10. Transparent nail polish

11. Mouse anti-PAX7 antibody (Santa Cruz, catalog number: sc-81648, storage temperature: $4^{\circ} \mathrm{C}$ )

12. Rabbit anti-MYOD antibody (Thermo Fisher Scientific, catalog number: PA5-23078, storage temperature: $4^{\circ} \mathrm{C}$ )

13. Alexa Fluor 488 goat anti-mouse IgG antibody (Thermo Fisher Scientific, catalog number: A11001 , storage temperature: $4^{\circ} \mathrm{C}$ )

14. Alexa Fluor 546 goat anti-rabbit IgG antibody (Thermo Fisher Scientific, catalog number: A11035 , storage temperature: $4^{\circ} \mathrm{C}$ )

15. Type I collagenase (Worthington, catalog number: CLS1, storage temperature: $4^{\circ} \mathrm{C}$ )

16. Culture and washing medium (see Recipes)

17. Digestion medium (see Recipes)

18. $5 \%$ BSA solution (see Recipes)

19. $2 \%$ PFA (see Recipes)

20. Washing solution for immunostaining (see Recipes)

21. Permeabilizing solution (see Recipes)

22. Blocking solution (see Recipes)

\section{Equipment}

1. Stereomicroscope (Zeiss, model: Stemi 2000-C)

2. Fluorescence microscope (Olympus, model: IX83 two-deck system)

3. Digital camera for fluorescence microscope (Olympus, model: DP80)

4. Water bath (AS ONE, model: TR-1A)

5. $\mathrm{CO}_{2}$ incubator (Eppendorf, model: Galaxy $170 \mathrm{~S}$ )

6. Tissue culture hood

\section{Software}

1. cellSens Imaging Software (Olympus)

2. Photoshop software (Adobe) 


\section{Procedure}

A. Preparation before dissection

1. Prepare the materials needed for dissection

a. Using a diamond pen, inscribe circles on glass Pasteur pipettes.

b. Prepare the pipette openings with diameters of $1 \mathrm{~mm}$ (small) or $5 \mathrm{~mm}$ (large). For creating the large pipette, break the pipette at the circle (Figure 1).

c. Heat-polish the cut ends of the Pasteur pipettes (both small and large) with a burner to prevent injuries to myofibers (Figure 1).

d. Use a large pipette to disaggregate the EDL muscle bundle to individual myofibers.

e. Then, use a small pipette to pick up and collect individual myofibers.

f. Fit heat-polished pipettes with rubber bulbs
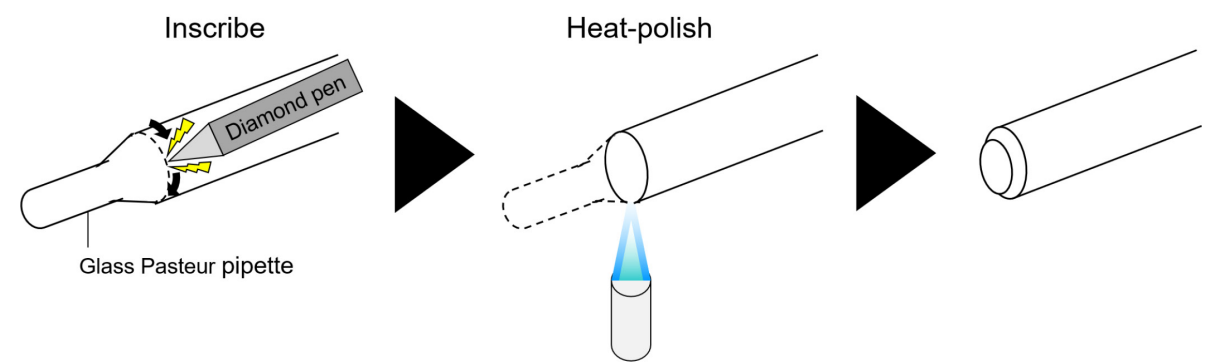

Figure 1. Workflow of making heat-polished pipettes (for large pipette).

2. Prepare the disaggregation medium

a. Immediately before dissection, prepare $1 \mathrm{~mL}$ of $0.2 \%$ type I collagenase solution in DMEM containing $1 \%$ PS per EDL muscle into a $7 \mathrm{~mL}$ Bijou sample container.

b. Sterilize the collagenase solution using a $0.45 \mu \mathrm{m}$ syringe filter.

c. Place the collagenase solution in a $\mathrm{CO}_{2}$ incubator at $37^{\circ} \mathrm{C}$.

3. Prepare $5 \%$ BSA solution

a. Sterilize the BSA solution using a $0.45 \mu \mathrm{m}$ syringe filter into a $25 \mathrm{~mL}$ self-standing tube.

b. Inactivate the BSA solution at $60^{\circ} \mathrm{C}$ for $30 \mathrm{~min}$ in a water bath.

c. Rinse the surface of all materials, including tubes, pipettes, and dishes with $5 \%$ BSA solution to prevent isolated individual myofibers from adhering.

4. Prepare DMEM containing $1 \%$ PS for culture and washing of myofibers.

5. Add 6-8 $\mathrm{mL}$ of DMEM containing $1 \% \mathrm{PS}$ in each deep Petri dish and place in a $\mathrm{CO}_{2}$ incubator at $37^{\circ} \mathrm{C}$ before use.

6. Prepare slide glasses enclosed on surface with pap-pen for immunostaining.

B. Digestion of EDL muscle bundle to individual myofibers (Figure 2)

1. Sacrifice 8-weeks-old C57BL/6 mice using cervical dislocation.

2. Spray hindlimbs with $70 \%$ ethanol and remove the skin of the hindlimb. 
3. Using needles, pin both forelimb palm on a dissection board and cut the distal tendons of the EDL and tibialis anterior (TA) muscles.

4. Gently pull both the distal tendons of the EDL and TA muscles by tweezers toward the proximal direction.

5. Remove the TA muscle.

6. Expose the EDL muscle by removing connective tissues on the proximal tendon of EDL muscle.

7. Carefully cut the proximal tendon of the EDL muscle, using a scalpel when the tendon is sufficiently exposed.

8. Place the isolated EDL muscle into a $7 \mathrm{~mL}$ Bijou sample container containing prewarmed digestion medium and incubate for $1.5 \mathrm{~h}$ at $37^{\circ} \mathrm{C}$ under $5 \% \mathrm{CO}_{2}$, gently shaking the tube every $15 \mathrm{~min}$.

Note: The appropriate digestion time depends on the size of the muscle and the enzymatic activity of collagenase. When the muscle becomes slightly swollen, and several individual myofibers are separated from the edge of the muscle, digestion should be completed.

9. Repeat the same procedure for the other hindlimb if necessary.

Note: Control intact and damaged myofibers should be used from the same mouse.

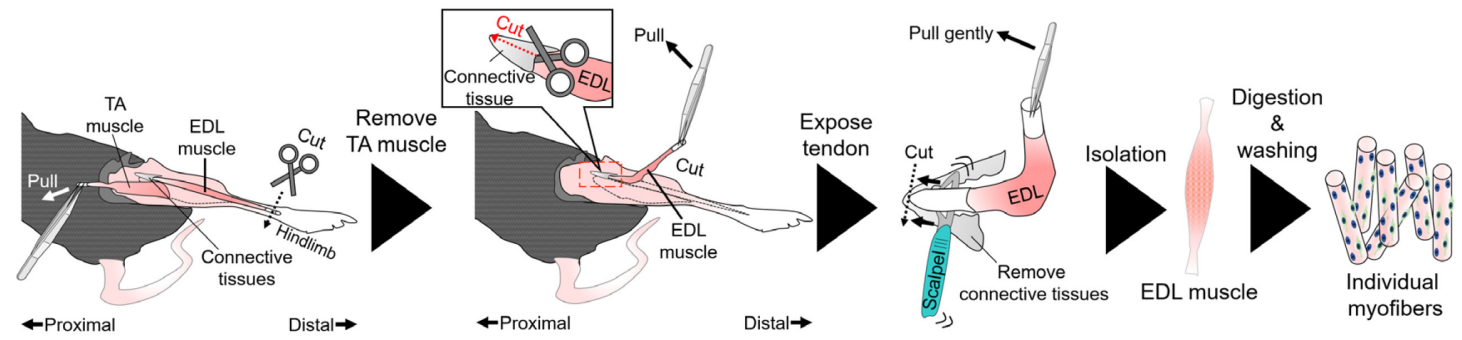

Figure 2. Workflow of dissection of mice to isolate myofibers.

C. Washing and collection of individual myofibers

All procedures should be performed using a stereo microscope in a tissue culture hood.

1. Place the digested EDL muscles into the Petri dish filled with washing medium.

2. Dissociate the muscle into individual myofibers using a large Pasteur pipette (pre-rinsed with $5 \%$ BSA) to triturate the muscle several times until it disaggregates.

3. To further dissociate myofiber bundles into individual myofibers, triturate the bundles 2-3 times using a small Pasteur pipette (pre-rinsed with 5\% BSA).

4. Remove the hypercontracted myofibers, tendons, vessels, nerves, and other debris, by washing with washing medium. The number of washes depends on the amount of debris.

5. Place individual myofibers in DMEM for $3 \mathrm{~h}$ at $37^{\circ} \mathrm{C}$ under $5 \% \mathrm{CO}_{2}$ to eliminate dying hypercontracted myofibers (the $2^{\text {nd }}$ incubation).

Note: The $2^{\text {nd }}$ incubation is important to obtain healthy undamaged myofibers. Damaged myofibers become hypercontracted during the incubation. 
Please cite this article as: Tsuchiya, Y. and Ono, Y. (2022). An in vitro Mechanical Damage Model of Isolated Myofibers in a Floating Culture Condition

D. Co-culture of intact myofibers with damaged myofibers (Figures 3-4)

All procedures should be performed using a stereo microscope in a tissue culture hood.

1. Add DMEM into each well of a 6 -well plate pre-coated with $5 \%$ BSA.

2. Transfer 150 intact individual myofibers per well using a small Pasteur pipette.

3. To remove the factors derived from damaged myofibers, aspirate DMEM using a small Pasteur pipette, and add fresh DMEM. Repeat this procedure several times. Do not aspirate DMEM completely not to dry myofibers. The volume of DMEM is determined as the ratio of 50 myofibers per $\mathrm{mL}$.

4. Collect 75 myofibers on the right side of the well using a small Pasteur pipette (Figure 3A).

5. Apply mechanical damage directly to the collected myofibers by rolling the edge of a new Pasteur pipette (coated with 5\% BSA and non-heat-polished) at least three times in the well (Figures 3B-3D).

6. After applying mechanical damage to myofibers, damaged myofibers should shrink immediately, presenting a hypercontracted morphology (Figure 3E).

Note: The Pasteur pipette is detached from damaged myofibers immediately after adding mechanical damage to the myofibers, because hyper-contracting damaged myofibers become sticky and coil easily around the pipette.

7. Confirm that the ratio of intact (undamaged) myofibers to damaged myofibers is $1: 1$ using a stereo microscope. The total number of intact myofibers used as a control condition (non-coculture damaged) must be equivalent to those in the damaged condition.

Note: Myofibers tend to spread elsewhere during the damage process (Figures 3B-3D). In this case, the spread myofibers are re-collected and damaged again.

8. To relocate damaged myofibers between intact myofibers, flush the medium with a small Pasteur pipette (Figure 3F). If hypercontracted myofibers are found in the control condition, remove them as soon as possible to minimize the influence of DMDFs on the intact healthy myofibers.

Note: In the undamaged control condition, aspiration of the culture medium should be minimized when damaged myofibers are washed out. 

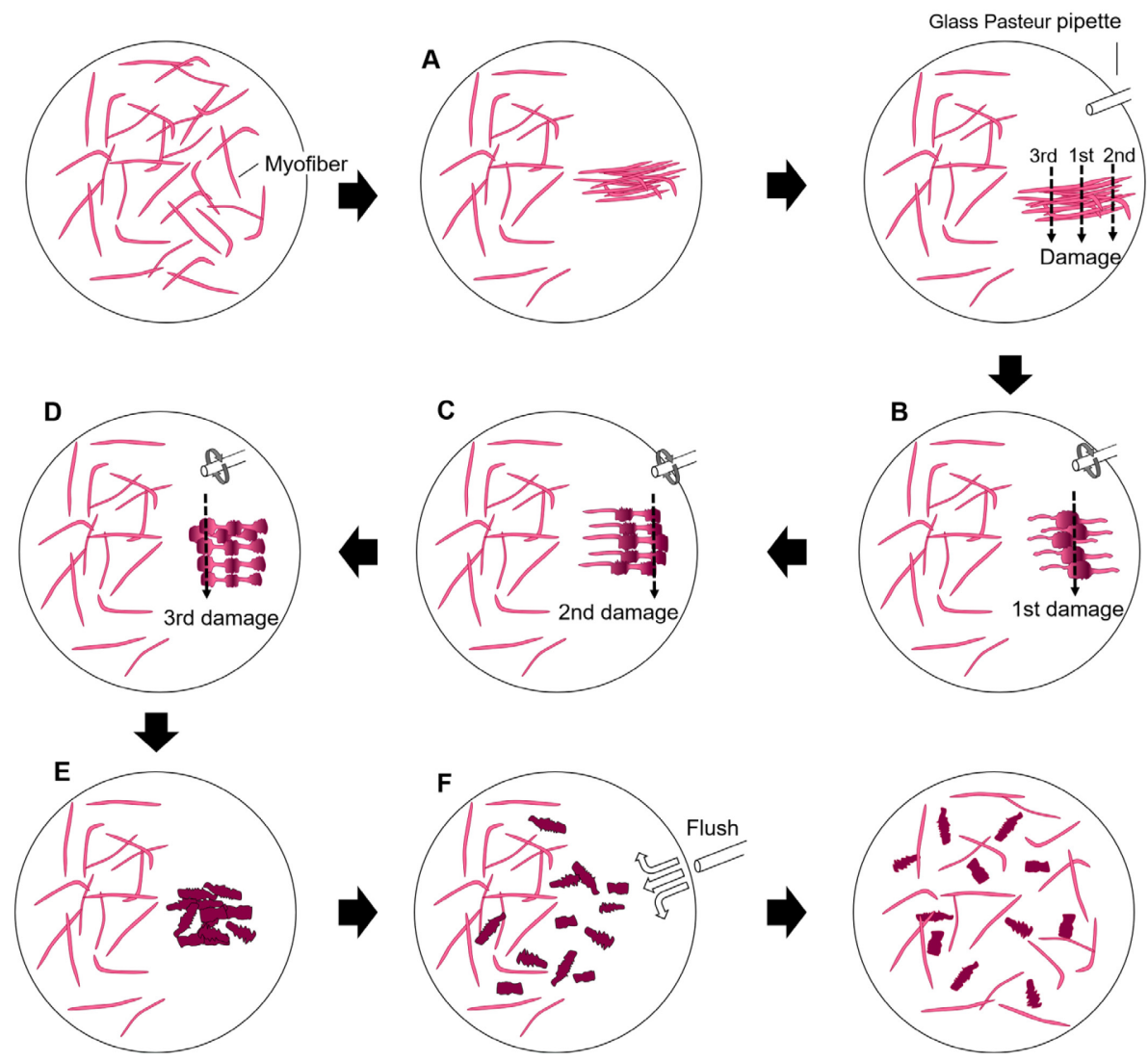

Figure 3. Workflow of co-culture of intact myofibers with damaged myofibers in a floating condition.

Culture of intact myofibers

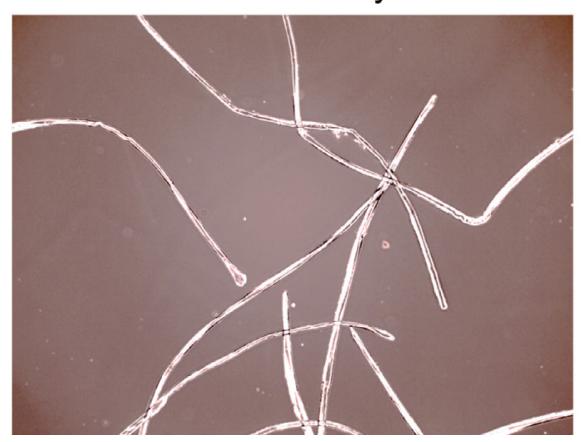

Co-culture of intact myofibers with damaged myofibers

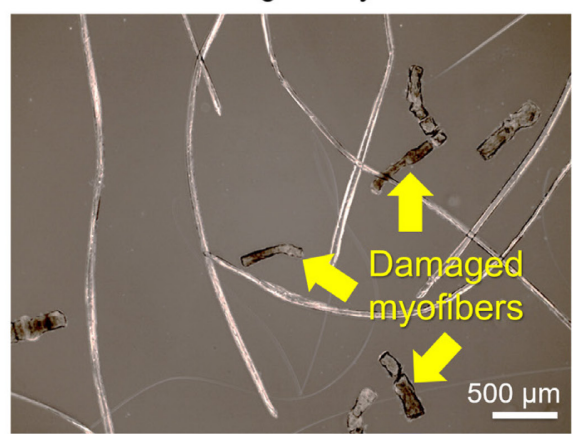

Figure 4. Representative images of undamaged (left) and damaged (right) conditions.

E. Immunostaining (Figures 5-6)

The stereo microscope should be used during manipulation of myofibers.

1. Coat the inside of $1.5 \mathrm{~mL}$ tubes and a small Pasteur pipette with $5 \% \mathrm{BSA}$ to prevent myofibers from adhering.

2. Transfer the myofibers into a $1.5 \mathrm{~mL}$ tube using a small Pasteur pipette and remove the excess amount of medium. 
Note: Transferred myofibers fall down to the bottom of the tube within $5 \mathrm{~min}$.

3. To fix the myofibers, add $2 \%$ PFA in PBS to the $1.5 \mathrm{~mL}$ tube containing myofibers. The final concentration of PFA would be less than $2 \%$.

4. Wash the myofibers three times with a washing solution (PBS-T: $0.025 \%$ Tween 20 in PBS). After myofibers are all gathered to the bottom, the washing solution is carefully replaced with a small Pasteur pipette.

5. Permeabilize with a permeabilization solution ( $0.3 \%$ Triton $\mathrm{X} 100$ in PBS) for 20 min at room temperature $\left(15-25^{\circ} \mathrm{C}\right)$.

6. Wash once with washing solution.

7. Block with a blocking solution ( $5 \%$ goat serum in PBS-T) for 20 min at room temperature (15$\left.25^{\circ} \mathrm{C}\right)$.

8. Wash three times with washing solution.

9. Incubate with appropriate primary antibodies diluted in PBS-T at $4^{\circ} \mathrm{C}$ overnight.

10. Wash three times with washing solution.

11. Incubate with the appropriate species-specific Alexa Fluor 488 and/or 546 fluorescencesecondary antibodies in PBS-T for 60 min at room temperature $\left(15-25^{\circ} \mathrm{C}\right)$.

12. Wash the cells twice with PBS-T and then wash once with PBS, to promote the attachment of myofibers onto the glass slide.

13. Enclose the surface at the edge with a pap-pen, to prevent myofibers from leaking out from the glass slide, and transfer the myofibers to the glass slide.

14. Remove the excess amount of PBS from the surface of the slide glass.

15. Mount the cover glass onto the slide glass with a drop $(\sim 50 \mu \mathrm{L})$ of mountain medium containing DAPI.

16. Stick the cover glass and the slide glass together with transparent nail polish at the four corners.

17. Image the samples under fluorescence microscope and analyze using the cellSens software

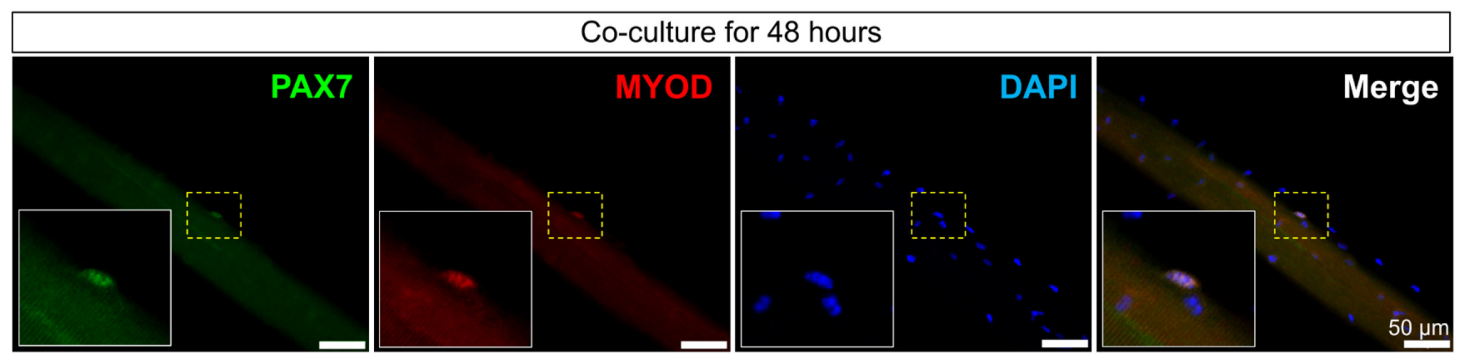

Figure 5. Representative immunofluorescence images of an activated satellite cell $\left(\mathrm{PAX7}^{+} / \mathrm{MYOD}^{+}\right)$associated with an intact healthy myofiber co-cultured with damaged myofibers for $48 \mathrm{~h}$. Scale bars, $50 \mu \mathrm{m}$. 
Please cite this article as: Tsuchiya, Y. and Ono, Y. (2022). An in vitro Mechanical Damage Model of Isolated Myofibers in a Floating Culture Condition.

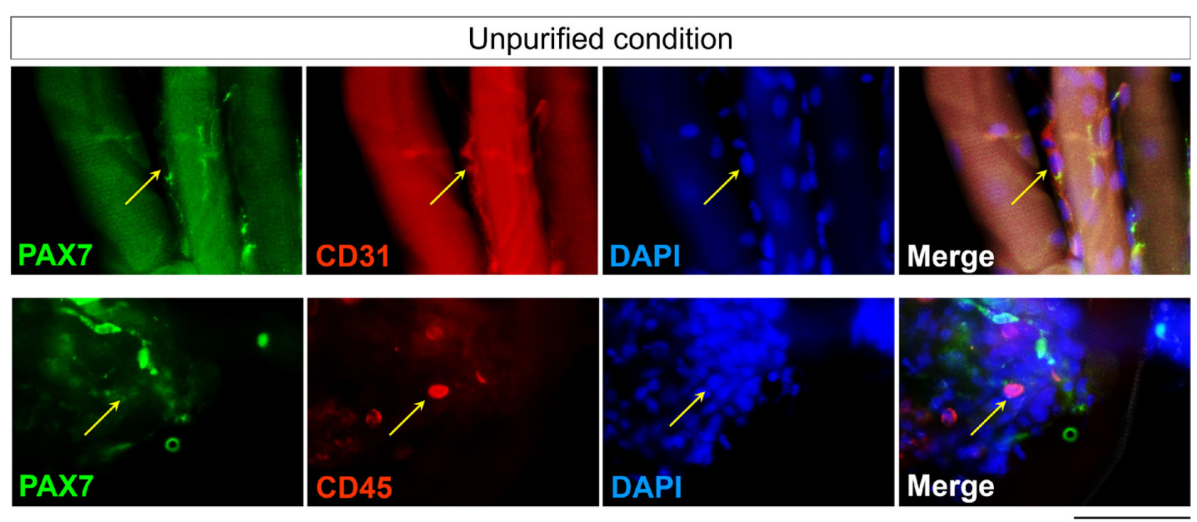

Figure 6. Representative immunofluorescence images of contamination of interstitial cells $\left(\mathrm{CD} 31^{+}\right.$or $\left.\mathrm{CD}^{+} 5^{+}\right)$associated with intact healthy myofibers in the unpurified condition. Scale bars, $50 \mu \mathrm{m}$.

\section{Data analysis}

Satellite cells associated with myofibers were visualized by immunostaining for PAX7 and MYOD proteins. $\mathrm{PAX} 7^{+} / \mathrm{MYOD}^{-}, \mathrm{PAX7}^{+} / \mathrm{MYOD}^{+}$, and $\mathrm{PAX7}-/ \mathrm{MYOD}^{+}$cell populations indicated quiescent, activated, and differentiated cells, respectively. Twenty individual myofibers were counted from each mouse, and the average number of 20 individual myofibers was used as one sample. When comparing the control (undamaged intact myofibers) and co-culture with damaged myofiber conditions, we used a paired Student's $t$-test (Tsuchiya et al., 2020).

\section{Recipes}

1. Culture and washing medium

DMEM supplemented with $1 \%$ PS.

Store at $4^{\circ} \mathrm{C}$.

2. Digestion medium

DMEM supplemented with $1 \%$ PS and $0.2 \%$ collagenase type 1 .

Sterilize using a sterile syringe filter $(0.45 \mu \mathrm{m}$ pore size $)$.

3. $5 \%$ BSA solution

PBS supplemented with 5\% BSA.

Inactivate the BSA solution for $30 \mathrm{~min}$ at $60^{\circ} \mathrm{C}$ by water bath.

Sterilize using a syringe filter $(0.45 \mu \mathrm{m}$ pore size $)$.

4. $2 \%$ PFA

Dilute 4\% PFA in PBS with the equivalent volume of PBS to obtain a final concentration of $2 \%$ PFA in PBS.

5. Washing solution to fixed myofibers (PBS-T).

PBS supplemented with $0.025 \%$ Tween 20 . 
Store at room temperature.

6. Permeabilizing solution

PBS supplemented with $0.3 \%$ Triton X100.

Store at room temperature.

7. Blocking solution

PBS-T supplemented with $5 \%$ goat serum.

\section{Acknowledgments}

This work was supported by the Japan Agency for Medical Research and Development (AMED, JP19bm0704036), JST FOREST Program (JPMJFR205C) and Grant-in-Aid for Scientific Research KAKENHI (17K13138 and 18K19749). This protocol was adapted from a previous study (Tsuchiya et al., 2020).

\section{Competing interests}

The authors declare no conflict of interest.

\section{Ethics}

This protocol for animal care and use was approved by the Ethical Committee (no. 1703161367) of Nagasaki University and Kumamoto University (A30-098).

\section{References}

1. Bischoff, R. (1986). A satellite cell mitogen from crushed adult muscle. Dev Biol 115(1): 140147.

2. Evano, B. and Tajbakhsh, S. (2018). Skeletal muscle stem cells in comfort and stress. NPJ Regen Med 3: 24.

3. Haugk, K. L., Roeder, R. A., Garber, M. J. and Schelling, G. T. (1995). Regulation of muscle cell proliferation by extracts from crushed muscle. J Anim Sci 73(7): 1972-1981.

4. Kardami, E., Spector, D. and Strohman, R. C. (1985). Selected muscle and nerve extracts contain an activity which stimulates myoblast proliferation and which is distinct from transferrin. Dev Biol 112(2): 353-358.

5. Li, Y. P. (2003). TNF-alpha is a mitogen in skeletal muscle. Am J Physiol Cell Physiol 285(2): C370-376.

6. Mauro, A. (1961). Satellite cell of skeletal muscle fibers. J Biophys Biochem Cytol 9: 493-495. 
7. Mezzogiorno, A., Coletta, M., Zani, B. M., Cossu, G. and Molinaro, M. (1993). Paracrine stimulation of senescent satellite cell proliferation by factors released by muscle or myotubes from young mice. Mech Ageing Dev 70(1-2): 35-44.

8. Ono, Y., Calhabeu, F., Morgan, J. E., Katagiri, T., Amthor, H. and Zammit, P. S. (2011). BMP signalling permits population expansion by preventing premature myogenic differentiation in muscle satellite cells. Cell Death Differ 18(2): 222-234.

9. Tatsumi, R., Anderson, J. E., Nevoret, C. J., Halevy, O. and Allen, R. E. (1998). HGF/SF is present in normal adult skeletal muscle and is capable of activating satellite cells. Dev Biol 194(1): 114-128.

10. Tsuchiya, Y., Kitajima, Y., Masumoto, H. and Ono, Y. (2020). Damaged Myofiber-Derived Metabolic Enzymes Act as Activators of Muscle Satellite Cells. Stem Cell Reports 15(4): 926940.

11. Vandenburgh, H. H., Sheff, M. F. and Zacks, S. I. (1984). Soluble age-related factors from skeletal muscle which influence muscle development. Exp Cell Res 153(2): 389-401.

12. Zammit, P. S., Golding, J. P., Nagata, Y., Hudon, V., Partridge, T. A. and Beauchamp, J. R. (2004). Muscle satellite cells adopt divergent fates: a mechanism for self-renewal? J Cell Biol 166(3): 347-357.

13. Zeng, L., Akasaki, Y., Sato, K., Ouchi, N., Izumiya, Y. and Walsh, K. (2010). Insulin-like 6 is induced by muscle injury and functions as a regenerative factor. $J$ Biol Chem 285(46): 3606036069. 